\title{
CONTROL DEL BARRENADOR DE LAS SEMILLAS, BEPHRATELLOIDES CUBENSIS ASHMEAD (HYMENOPTERA: EURYTOMIDAE) EN GUANÁBANA, ANNONA MURICATA L. (ANNONALES: ANNONACEAE)
}

\author{
Luis M. Hernández-Fuentes'1, Néstor BAUTISTA-MARTínez', \\ José L. CARrIllo-SÁnCHeZ ${ }^{1}$, Hussein SÁNChEZ ARROYO1, \\ Mario A. URías-López ${ }^{2}$ y Manuel D. SAlas ARAIZA ${ }^{3}$
}

1Entomología y Acarología. Campus Montecillo. Colegio de Postgraduados.

C.P. 56230. Montecillo, Texcoco, Edo. de México.

2INIFAP-Campo Experimental Santiago Ixcuintla. C.P. 63300.

Santiago Ixcuintla, Nayarit, MÉXICO.

1mhf01@yahoo.com.mx. 3Universidad de Guanajuato. Instituto de Ciencias Agrícolas.

Ex. Hacienda El Copal Carr. Irapuato-León Km. 5. Irapuato, Guanajuato C.P. 36500.

\section{RESUMEN}

En condiciones de campo se evaluaron los insecticidas dimetoato, malatión, clorpirifos-etil, cipermetrina, endosulfán y azadiractina para el control del barrenador de las semillas $B$. cubensis en guanábana. Las dosis aplicadas fueron: 40, 150, 48, 40, 94. 5 y $30 \mathrm{~g}$ de i.a. en $100 \mathrm{~L}$ de agua, respectivamente; asimismo, se evaluó el embolsado de frutos con tela de organza y bolsas de plástico transparentes y perforadas para evitar la acumulación de humedad. El experimento se repitió en dos ocasiones. La variable evaluada fue el porcentaje de semillas infestadas. El embolsado de frutos con tela de organza fue el mejor tratamiento ( 0.0 y $1.6 \%$ de infestación), en los frutos cubiertos con bolsas de plástico se observó cero porciento de infestación, pero éstos presentaron quemaduras y una disminución en el crecimiento. De los insecticidas evaluados el dimetoato fue el mejor tratamiento $(0.75$ y $5.9 \%$ de infestación) seguido de la cipermetrina (0.8 y $17.83 \%$ de infestación).

Palabras clave: Bephratelloides cubensis, guanábana, control.

\section{ABSTRACT}

The insecticides dimethoate, malathion, ethyl-chlorpyriphos, cipermetrine, endosulphan and azadirachtine were evaluated for controlling the seed borer B. cubensis on soursop under field conditions. Dosage of 40, 150, 48, 40, 94.5 and $30 \mathrm{~g}$ of active ingredient in $100 \mathrm{~L}$ of water, respectively, were used. Likewise, pocketing of fruits with organdi mesh and perforated, transparent plastic bags to avoid humidity accumulation were evaluated. The experiment was repeated twice. The evaluated variable was percentage of infested seeds. Pocketing of fruits with organdi mesh was the best treatment ( 0.0 and $1.6 \%$ of infestation). The fruits covered with plastic bags showed zero percent of infestation, but these displayed burn spots and a decrease in growth. Dimethoate was the best insecticide treatment ( 0.75 and $5.9 \%$ of damage), followed by cipermetrine ( 0.8 and $17.83 \%$ of damage).

Key words: Bephratelloides cubensis, soursop, control. 


\section{INTRODUCCIÓN}

El guanábano (Annona muricata L.) es nativo de América y uno de los frutales más apreciados en los trópicos de Centro y Sudamérica; es un frutal con gran potencial económico, dado su valor comercial y la demanda en el mercado externo; sin embargo, su extensión y cultivo se han limitado debido principalmente a la baja producción de frutos y al ataque de plagas y enfermedades (Coto \& Saunders 2001; Cruz et al.2002). Vidal \& Nieto (1997) reportan para el cultivo de la guanábana en México una superficie de 5,915 ha. El estado de Nayarit es de los principales productores con 1,814 ha sembradas (comunicación personal) ${ }^{1}$. El barrenador de las semillas Bephratelloides cubensis Ashmead, es la plaga principal de las anonáceas cultivadas (Peña et al. 1984, 2002). Velasco \& Rodríguez (1980) reportaron la presencia de B. cubensis en Nayarit y Rodríguez et al. (1980) consignan $60 \%$ de frutos atacados. La hembra inserta los huevos en semillas de frutos de guanábana cuando éste mide de 3.1 a $7.6 \mathrm{~cm}$ de diámetro (Hernández et al. 2006) y de 3 a $4 \mathrm{~cm}$ en atemoya (Annona cherimolla x A. squamosa), al eclosionar, la larva se alimenta del endospermo; pupa dentro de la semilla y al emerger el adulto realiza una perforación hasta la superficie del fruto (Nadel \& Peña 1991). El mayor daño causado por este insecto es indirecto, debido a que el adulto al emerger deja una entrada por donde atacan fitopatógenos. Al respecto Nadel \& Peña (1991) reportan un incremento de hasta cuatro veces el daño por enfermedades, por lo que lo más importante es evitar que la hembra oviposite. Algunos productores de guanábana en Nayarit realizan hasta seis aplicaciones de plaguicidas por año para controlar al barrenador, sin lograr un control efectivo. Es por ello que el objetivo de este estudio fue evaluar diferentes insecticidas y barreras físicas como alternativas en el manejo del barrenador.

\section{MATERIALES Y MÉTODOS}

\section{Ubicación del experimento}

Las actividades se realizaron en una plantación de guanábana de 4 ha ubicada en la comunidad de Las Varas, Compostela, Nayarit, México, a los 21¹1'15.9” de latitud Norte y 105'8'2.9" de longitud Oeste, a $40 \mathrm{msnm}$. Los árboles de guanaban tenían un porte de $2.5 \mathrm{~m}$ de altura en promedio. Se realizaron dos evaluaciones, una por cada ciclo de producción. La primera se realizó en el periodo de producción de marzo-junio y la segunda de julio-octubre de 2006, con una temperatura y humedad relativa media mensual de $26.97{ }^{\circ} \mathrm{C}$ y $59.49 \%, 29.33{ }^{\circ} \mathrm{C}$ y $69.17 \%$, respectivamente. La metodología, descrita posteriormente, fue prácticamente la misma en las dos evaluaciones, realizando ligeras variaciones en la segunda evaluación con base en la experiencia de la primera. 


\section{Diseño experimental}

El diseño experimental fue completamente al azar. La variable respuesta fue el porcentaje de semillas infestadas. Se realizaron 4 repeticiones por tratamiento, cada repetición consistió de un árbol; de cada uno de éstos se cortaron cinco frutos, para dar un total de 20 en cada tratamiento. A éstos se les extrajeron las semillas en etapa próxima a cosecha y se contabilizaron aquellas sanas e infestadas. Se consideró semilla infestada aquella de la cual ya había emergido el adulto o que al disectarla se encontrara al insecto en cualquiera de sus estados biológicos. Se realizó un análisis de varianza con el modelo lineal general para determinar diferencias entre tratamientos y posteriormente realizar una comparación de medias de éstos (Tukey, $\cdot=0.05$ ). Previamente se determinó homogeneidad de varianza de las observaciones para cada tratamiento con la prueba de Hovtest (Levene's test) (Snedecor y Cochran, 1989) en SAS $\odot$ versión 8.1 (2000).

\section{Tratamientos}

Primera evaluación. En el caso de guanábana, en México no se tienen plaguicidas registrados en CICOPLAFEST (Comisión Intersecretarial para el Control de Plaguicidas, Fertilizantes y Sustancias Tóxicas) y COFEPRIS (Comisión Federal para la Protección Contra Riesgos Sanitarios). Sin embargo, los productores de la región de estudio utilizan diferentes insecticidas para el control del barrenador. Entre los principales están paratión metílico, dimetoato y clorpfirifos-etil. Para este estudio, se tomaron como referencia producto y dosis autorizadas para frutales tropicales, como mango y naranja. Se aplicaron los insecticidas dimetoato, malatión, clorpirifos-etil, cipermetrina y endosulfán, en dosis de 40, 150, 48, 40 y $94.5 \mathrm{~g}$ de i.a. en $100 \mathrm{~L}$ de agua, respectivamente. Se incluyó un testigo al que sólo se le aplicó agua. Como barreras físicas, se utilizaron bolsas de plástico transparente con ocho aberturas de $1 \mathrm{~cm}$ de largo, aproximadamente, para evitar la acumulación de humedad y bolsas de tela de organza de 372 perforaciones por pulgada ${ }^{2}$. Las bolsas de plástico y tela se retiraron a los 49 días después de ser colocadas.

Segunda evaluación. Debido a que en la primera evaluación se observaron daños al fruto por quemaduras de sol con el uso de bolsas de plástico, para esta evaluación no se consideró este tratamiento. Los tratamientos con insecticidas fueron: malatión, dimetoato, cipermetrina y clorpirifos-etil, a las mismas dosis que en la primera evaluación. También se evaluó azadiractina en dosis de $0.3 \mathrm{~g}$ de i.a. por L de agua. Como barrera física sólo se evalúo tela de organza. De la misma forma se tuvo un testigo al que sólo se le aplicó agua.

\section{Selección de frutos y aplicación de insecticidas}

Primera evaluación. Para evitar que los frutos fueran dañados por el barrenador antes de ser cubiertos con tela de organza, bolsas de plástico o ser 
aplicados con insecticidas, se cubrieron aquellos que medían entre 2 y $3 \mathrm{~cm}$ de diámetro. Para homogeneizar tamaños se eliminaron del árbol los frutos con menor y mayor tamaño del elegido. En el caso de los tratamientos con insecticidas, cuando los frutos median entre 3 y $4 \mathrm{~cm}$ de diámetro (9 días después del embolsado con tela), se descubrieron y al día siguiente se realizó la primera aplicación. Para identificar aquellos frutos que fueron cubiertos y tratados con insecticidas se marcaron con esmalte. Debido a la homogeneidad en el tamaño de los frutos y a que el insecto daña frutos de $7 \mathrm{~cm}$ o menos de diámetro (Hernández et al. 2006), sólo se hicieron dos aplicaciones; la primera fue el 24 de marzo y la segunda el 8 de abril. En este lapso los frutos alcanzaron un tamaño mayor al susceptible. Se utilizó una aspersora manual de mochila de 87 libras por pulgada cuadrada de presión y se aplicó a todo el árbol con $0.625 \mathrm{~L}$. Aunque Peña et al. (1984) recomiendan realizar aplicaciones de plaguicidas para el control de $B$. cubensis en atemoya entre las 15 y $16 \mathrm{~h}$; las aplicaciones se realizaron alrededor de las $12 \mathrm{~h}$, tiempo del Pacífico, ya que en este momento es cuando la hembra tiene mayor actividad de vuelo en busca de frutos y oviposición (Hernández et al. 2006). La disección de los frutos seleccionados se realizó a los 63 y 64 días después de la primera aplicación.

Segunda evaluación. A diferencia de la primera evaluación, donde se eliminaron frutos para homogeneizar diámetros y cubrirse con tela hasta que alcanzaran el tamaño más susceptible, en esta segunda evaluación sólo se protegieron aquellos frutos de $2.5 \mathrm{~cm}$ de diámetro para evaluar el tratamiento con tela de organza, el resto de los frutos, a los cuales se les aplicarían los insecticidas, se dejaron descubiertos. Debido a la heterogeneidad en el tamaño de los frutos y con el objetivo de aplicar los insecticidas de cada tratamiento en todos aquellos frutos susceptibles, se realizó una aplicación cada 10 días, en total se realizaron tres aplicaciones. La primera aplicación se realizó el 9 de agosto cuando se observaron frutos de $3.1 \mathrm{~cm}$ de diámetro. El gasto de la mezcla fue de $0.635 \mathrm{~L}$ por árbol. La disección de los frutos se realizó a los 80 y 81 días después de la primera aplicación.

\section{RESULTADOS}

Se observó homogeneidad de varianza $(\alpha=0.05 ; \mathrm{p}=0.1414$ y 0.4885 , primera y segunda evaluación, respectivamente) para los tratamientos evaluados. El análisis de varianza indicó diferencias entre tratamientos $(\alpha=0.05 ; \mathrm{p}=0.0001$ y 0.0049 , primera y segunda evaluación, respectivamente).

Con base en el análisis estadístico, los mejores tratamientos en la primera evaluación fueron el uso de bolsas de plástico y de organza, siguiéndoles el dimetoato, cipermetrina, malatión y endosulfán, estos dos últimos sin diferencia 
estadística respecto al testigo (Figura 1A). En la segunda evaluación se confirmó una menor infestación de los frutos cubiertos con tela de organza y los tratados con dimetoato y cipermetrina; con diferencia estadística respecto al testigo y a la azadiractina, excepto el tratamiento con cipermetrina (Figura 1B).

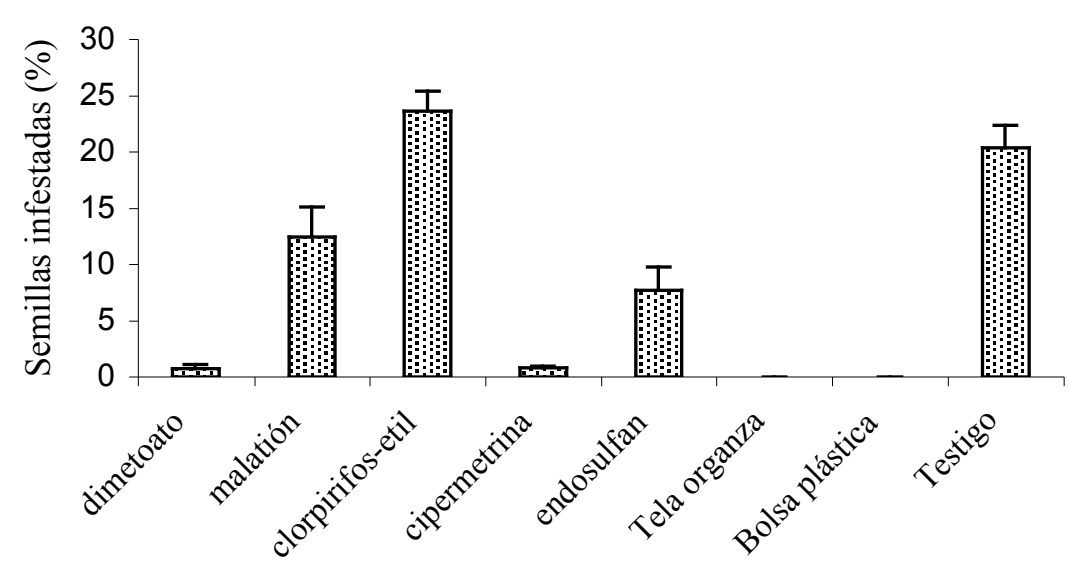

A

B

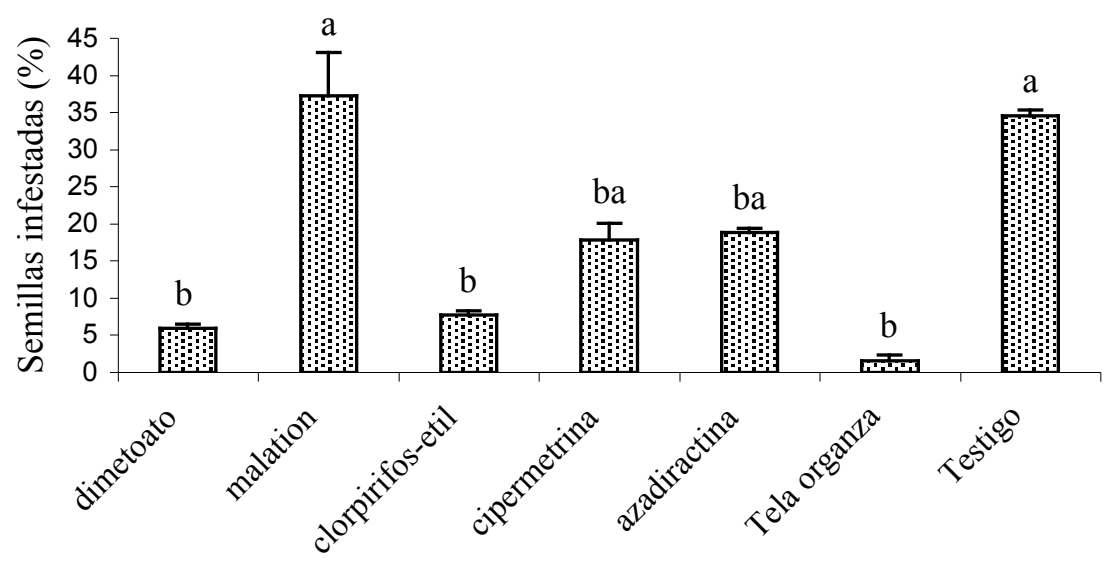

Figura 1

Infestación de guanábana por B. cubensis. Las Varas, Compostela, Nayarit, México. 2006. A y B, primera y segunda evaluación, respectivamente. Tratamiento con diferente letra es significativamente distinta (Tukey, $\alpha=0.05$ ). Las barras superiores indican el error estándar. 


\section{DISCUSIÓN}

Aunque el uso de bolsa plástica evitó la infestación, los frutos mostraron quemaduras y disminución del crecimiento. Por ello, en la segunda evaluación no se consideró este tratamiento. Micheletti et al. (2001) evaluaron métodos químicos y barreras físicas (bolsa de plástico perforadas, cerradas y abiertas) para controlar Bephratelloides pomorun y Cerconota annonella en frutos de guanábana y concluyeron que con el uso de bolsas se obtiene el menor numero de perforaciones por fruto de B. pomorum. Estos autores no mencionan el color de las bolsas ni que haya habido daños por quemaduras de sol a los frutos. Por observación personal, los frutos que reciben mayor radiación solar adquieren un color verde claro y presentan desarrollo normal. El color de la bolsa puede ser importante y convendría realizar una evaluación de diferentes colores. Los frutos cubiertos con tela de organza presentaron menor infestación en las dos evaluaciones, en la primera evaluación algunos de los frutos (cinco del total) fueron dañados por pulgones y piojos harinosos durante los primeros días; sin embargo, sus depredadores penetraron por las perforaciones de la bolsa y se logró controlar el crecimiento de éstos. El desarrollo de piojos harinosos en frutos embolsados con tela es consignado por Peña et al. (2002); no obstante, se deduce que el tamaño de las perforaciones en la tela usada en esta investigación, permitió la entrada de depredadores. Peña \& Nagel (1988) evaluaron malatión y permetrina en condiciones de laboratorio con aplicaciones tópicas al adulto de $B$. cubensis y concluyeron que el malatión en dosis de $0.57 \%$ de i.a. $\mathrm{L}^{-1}$ fue mejor que la permetrina en dosis de $0.256 \%$; asimismo, en condiciones de campo evaluaron el promedio de semillas infestadas en frutos de atemoya aplicando malatión, permetrina y fenvalerato en dosis de $0.92,0.79$ y $0.143 \mathrm{~g}$ de i.a. L-1, respectivamente, sin observar diferencia estadística (Duncan, $=0.05$ ) entre los tratamientos. Sin embargo, con malatión obtuvieron la menor infestación (4.5 semillas por fruto). En el caso de guanábana, se ha observado que el malatión en dosis de $1 \mathrm{~g}$ de i.a. L-1 ocasiona fitotoxicidad, provocando caída prematura de flores y frutos de $2 \mathrm{~cm}$ o menos de diámetro (no publicado). En el guanábano se pueden observar diferentes tamaños de frutos e incluso flores simultáneamente, por lo que se debe valorar el momento de aplicación y la dosis de malatión.

El efecto de extracto de nim y azadiractina se ha evaluado en más de 200 especies de insectos plaga (Mordue \& Blackwell 1993); sin embargo, en himenópteros fitófagos sus usos como control químico son poco documentados. El uso de azadiractina como deterrente de la oviposición y repelencia se ha observado en Helicoverpa armigera, Spodoptera frugiperda y Lucilia sericata (Schmutterer 1990). Con azadiractina, se observó una disminución en la infestación de semillas por B. cubensis (Figura 1B); no obstante, no fue estadísticamente diferente respecto al testigo. En esta evaluación el clorpirifos-etil presentó un control similar 
al dimetoato, cipermetrina y tela de organza, la diferencia en el resultado de este tratamiento con respecto a la primera evaluación puede deberse a una tercera aplicación.

\section{AGRADECIMIENTOS}

Los autores agradecen a la Fundación Produce Nayarit A. C. y a la Junta Local de Sanidad Vegetal "Costa de Chila-Compostela" por el apoyo recibido para la realización de esta investigación.

\section{LITERATURA CITADA}

Coto, D. A. \& J. Saunders L. 2001. Insectos plaga de la guanábana (Annona muricata) en Costa Rica. Manejo Integrado de Plagas 61:60-68.

Cruz, C. J. G., P. A. Torres L., J. C. Delgado M., V. Domínguez M., D. Martínez P. \& O. Franco M. 2002. El Guanábano: Agronomía y usos de frutos tropicales. Universidad Autónoma Chapingo. Chapingo, México. 177 p.

Hernández F. L. M., N. Bautista M., J. L. Carrillo S., J. Cibrián T. \& M. A. Urías L. 2006. Bephratelloides cubensis: Comportamiento diurno y selección de frutos en guanábana (Annona muricata). Pp. 696-699. In: G. E. Estrada V., J. Romero N., A. Equihua M., C. Luna L. y J. L. Rosas A. (Eds). Entomología Mexicana Vol. 5. Sociedad Mexicana de Entomología A. C. Texcoco, México.

Micheletti, B. F. S. M., A. G. Santos de Melo A. G. V. Sousa B. e F. Leite Gomes. 2001. Controle de Cerconota anonella (Sepp) (Lep.: Oecophoridae) e de Bephratelloides pomorum (Fab.) (Hym.: Eurytomidae) em frutos de graviola (Annona muricata L.). Rev. Bras. Frutic. 23(3):722-725.

Mordue, J. A. \& A. Blackwell. 1993. Azadirachtin: an update. J. Insect. Physiol. 39(11):903924.

Nadel, H. \& J. Peña, E. 1991. Seasonal oviposition and emergence activity of Bephratelloides cubensis (Hymenoptera: Eurytomidae) a pest of Annona species in Florida. Enviromental Entomology 20(4):1053-1057.

Peña, J. E., H. Glenn, \& R. M. Baranowski. 1984. Important insect pest of Annona spp. in Florida. Proc. Fla. State Hort. Soc. 97:337-340.

Peña, J. E. \& Nagel J. 1988. Effectiveness of pesticides against two tropical fruit pests. Proc. Fla. State Hort. Soc. 101:249-251.

Peña, J. E., H. Nadel, M. Barbosa-Pereira \& D. Smith. 2002. Pollinators and pest for Annona species. Pp: 197-221. In: J. E. Peña L., J. L. Sharp. \& M. Wysoki (Eds). Tropical fruit pests and pollinators: biology, economic, natural enemies and control. CABI International Publishing.

Rodríguez, G. C., J. J. Velasco C. \& M. Velasco C. 1980. Producción de guanábano (Annona muricata) en el estado de Nayarit. Pp: 141-153. In: Memoria 3er. Simposium de la Investigación, Desarrollo Experimental y la Docencia en CONAFRUT Durante 1979. Tomo 1. CONAFRUT-SARH. Jalapa, Veracruz. 
Schumutterer, H. 1990. Properties and potential of natural pesticides from the neem tree, Azadirachta indica. Annu. Rev. Entomol. 35: 271-297.

Snedecor W. G. \& W. Cochran G. 1989. Statistical methods. 5ta. Edición. Iowa State University Press. Ames, Iowa. 503 p.

Statistical Analysis System SAS User's guide. 2000. Statistics version 8.1. SAS Institute Inc. Cary, North Caroline, USA.

Velasco, C. J. J. \& C. Rodríguez G. 1980. Producción de guanábano (Annona muricata) en el estado de Nayarit. Pp. 633-652. In: Memoria 3er. Simposium de la Investigación, Desarrollo Experimental y la Docencia en CONAFRUT Durante 1979. Tomo 2. CONAFRUT-SARH. Jalapa, Veracruz.

Vidal, H. L. \& D. Nieto A. 1997. Diagnóstico técnico y comercial de la guanábana en México. Pp. 1-17. In: Memoria: I Congreso Internacional de Anonáceas. 12-14 de noviembre de 1997. Universidad Autónoma Chapingo. Chapingo, México.

Recibido: 12 de enero de 2007

Aceptado: 20 de septiembre de 2007 